\title{
Editorial
}

\section{Harnack's Estimates: Positivity and Local Behavior of Degenerate and Singular Parabolic Equations}

\author{
Emmanuele DiBenedetto, Ugo Gianazza, Mikhail Safonov, \\ José Miguel Urbano, and Vincenzo Vespri
}

Received 20 November 2006; Accepted 28 November 2006

Copyright (c) 2007 Emmanuele DiBenedetto et al. This is an open access article distributed under the Creative Commons Attribution License, which permits unrestricted use, distribution, and reproduction in any medium, provided the original work is properly cited.

The subject of Harnack's inequalities and more generally of regularity estimates for degenerate and singular elliptic and parabolic equations has developed considerably in recent years, in many unexpected and challenging directions; therefore it seemed timely to trace an overview that would highlight emerging trends and issues of this fascinating research topic.

This special issue is an outgrowth of a Summer School, that took place in Cortona (Italy) in Summer 2005, and builds on the recent developments that were presented at that meeting and on the ones that followed soon after.

The editors took the opportunity kindly offered by the BVP editor-in-chief to record this momentous series of events into an issue that would emphasize and stress the importance of this kind of research, both in terms of its intrinsic results and the new techniques it offers in classical analysis. The relevance of Harnack's estimates has been brought to light by the recent Perelman's solution of the Poincaré conjecture, which ultimately relies on a Harnack estimate.

The editors aimed at a volume that might serve as a reference point in the field, and as a source of inspiration for young researchers, therefore collecting cutting-edge, original and unpublished research articles.

The opening contribution by Kassmann is a survey on the main results and applications of the Harnack inequality. The author focuses mainly on the analytic perspective, 
but comments on the geometric and probabilistic significance of Harnack's inequalities as well. The attention is mainly on classical results and an extensive and thorough list of references is given.

In Abdulla's paper, the author gives a criteria for existence, uniqueness, and comparison principle for the Dirichlet problem for the nonlinear parabolic equation

$$
u_{t}=\Delta u^{m}-b u^{\beta}
$$

posed in a noncylindrical space-time domain, reducing to a single point both at the initial and at the final times. Some generality is allowed in the shape of the domain near the final time. The conditions include relations between the exponents and the coefficient in the equation and, above all, assumptions on the boundary manifold. While technically, the contribution underscores the role of Wiener-type criteria in boundary regularity theory.

Bhattacharya proves a local boundary Harnack principle for $C^{2}$ domains for solutions of the $\Delta_{\infty}$ equation, extending results of a previous paper of his, where he had proved the same principle for domains with flat boundaries. Then he studies bounded $\infty$-harmonic functions which have boundary data 1 in a neighborhood of a point $x \in \partial \Omega$ and 0 otherwise,

$$
\begin{array}{ccc}
\Delta_{\infty} u^{r}=0 & \text { in } \Omega, \\
u^{r}=1 \quad \text { on } B_{r}(x) \cap \partial \Omega, & u^{r}=0 \quad \text { on } \partial \Omega \backslash B_{r}(x) .
\end{array}
$$

He shows existence of a maximal (minimal) solution and proves that

$$
u^{2 r} \leq C u^{r} \quad \text { on } \Omega \backslash B_{3 r}(x) .
$$

He obtains uniqueness of these solutions when $\Omega$ is a half space and $u^{r} \rightarrow 0$ at $\infty$. Finally, he gives some blow-up rates of singular solutions.

Biroli and Marchi investigate the validity of the elliptic Harnack inequality for Schrödinger-type operators defined by a class of strongly local, $p$-homogeneous Dirichlet forms and by potentials in suitable Kato classes. Similar results were obtained by Biroli in a previous paper for operators associated to subelliptic $p$-Laplacians, but the techniques used there do not seem to be easily adaptable to the more general case treated here and a different method has been applied. The main results obtained are local uniform estimates for local subsolutions, the Harnack inequality for positive local solutions and the continuity for local solutions. The paper is original, the generality of the operators considered is appealing and the method of proof, which cannot make any use of Moser's iteration technique, is interesting.

In their paper, Bonforte and Vazquez study local and global properties of positive solutions to the so-called fast diffusion equation, namely

$$
u_{t}-\Delta u^{m}=0
$$

when $m<1$. The paper collects and expands in a well-organized way some investigations previously started by the authors.

Ferrari and Salsa contributed a paper on elliptic PDEs in divergence form and its applications to free boundary problems. They establish for the first time for divergence-form 
equations with nonsmooth coefficients an analogue of a powerful tool previously introduced by Caffarelli for the Laplacian. This consists in constructing, from a solution, a new subsolution equal to the supremum of the solution itself over balls of variable radia. This is extremely useful to prove the $C^{1, \alpha}$ regularity of two-phase free boundaries. The authors carry this out in the context of their operators.

The paper by Harjulehto, Kinnunen, and Lukkari is concerned with the variable exponent $p$-Laplacian. The first result is a weak Harnack inequality for nonnegative supersolutions. With respect to known results, the new fact is that solutions are taken in $L^{p}$ spaces and are not necessarily bounded. This Harnack estimate is then used to prove that supersolutions are lower semicontinuous and to characterize the singular set in terms of a capacity, which is tailored on the variable exponent $p$ in a natural way. The method used to prove the Harnack estimate is the usual Moser iteration technique, but great care is applied to take into account the variable exponent.

Kogoj and Lanconelli prove Liouville-type theorems for second-order operators of the form

$$
L=\sum_{i, j}^{N} \partial_{x_{i}}\left(a_{i j}(x) \partial_{x_{j}}\right)+\sum_{i=1}^{N} b_{i}(x) \partial_{x_{i}}-\partial_{t},
$$

where $x \in \mathbb{R}^{N}$, all coefficients are smooth, the matrix $\left(a_{i j}(x)\right)$ is nonnegative definite at each $x$ and the first-order term is divergence free, that is, $\sum_{i=1}^{N} \partial_{x_{i}} b_{i}(x)=0$. There are assumptions about the homogeneity of $L$ with respect to the group of dilations

$$
\left(\lambda^{\sigma_{1}} x_{1}, \ldots, \lambda^{\sigma_{N}} x_{N}, \lambda^{2} t\right)
$$

with $1 \leq \sigma_{1} \leq \sigma_{2} \leq \cdots \leq \sigma_{N}$, which force the coefficients to be polynomials. If $p, q$ are polynomials and $u$ solves $L u=p$ with $u \geq q$ in $\mathbb{R}^{N+1}$, then $u$ is a polynomial provided $u(0, t)=O\left(t^{m}\right)$ as $t \rightarrow \infty$ for some $m$. In addition, the authors give estimates of the degree of $u$ in terms of the homogeneous degrees of $p$ and $q$. In the third part of the paper, the authors establish Liouville-type theorems of asymptotic type, that is, describing the behavior of $u$ at $\infty$, in half spaces. In this case they assume there exists a Lie structure over $\mathbb{R}^{N+1}$ under which $L$ is left invariant. Finally, these results are applied to several examples of operators.

Lewis and Vogel study overdetermined boundary conditions for positive solutions to some elliptic partial differential equations of $p$-Laplacian type in a bounded domain $D$. The authors show that these conditions imply uniform rectifiability of $\partial D$ and also that they yield the solution to certain symmetry problems. Despite the sophisticated hard analysis used by the authors, the paper is quite accessible.

Lieberman considers parabolic equations of the type

$$
u_{t}-\operatorname{div} a(x, t, u, D u)=b(x, t, u, D u) .
$$

The usual regularity and ellipticity conditions assumed on $a$ are relaxed. For instance, the author proves a priori estimates, in terms of the structure conditions on $a(x, t, u, D u)$, allowing for nonpolynomial growth conditions of the vector field $a$; the author is able to obtain results in case of the so-called exponential growth conditions: $a(\cdot, z)$ grows 
exponentially with $z$ (the gradient variable). Moreover, he is able to derive estimates on the solutions with a very weak assumption on the first derivatives of $a$ with respect to the gradient variable (actually all the assumptions are on $a$ itself); this is in turn plugged in Moser's iteration scheme to get the a priori gradient bounds. Many examples and variants are provided in Sections 6, 7, and 8. The techniques presented are novel and nontrivial.

It is well known that the components $F_{i}$ of a quasiregular mapping satisfy a secondorder quasilinear differential equation which is degenerate elliptic. Many results on quasiregular mappings are proved using the theory of such equations, that is, nonlinear potential theory, applied to the coefficients of $F$. The precise form of the equation satisfied by the $F_{i}$ 's is not important. In a previous paper, Martio, Miklyukov, and Vuorinen, together with D. Franke and R. Wisk, found a nice way to express the fact that a function $u$ satisfies an equation like the one satisfied by the components of a quasiregular mapping. They defined several classes of differential forms $w$ on a Riemannian manifold that they called $\mathcal{W} T$-classes. The goal of the present paper is to prove a removable singularity theorem for $\mathcal{W} T$-differential forms. As an application, one gets a corresponding removable singularity theorem for quasiregular mappings.

Safonov and Cho deal with linear second-order elliptic equations both in divergence and nondivergence forms, and prove global Hölder estimates for solutions to homogeneous Dirichlet conditions. In the paper, particular care is put in considering limit situations (e.g., for the parameters involved) or in giving simpler proofs for standard situations. The general structure of the paper clearly highlights what are the main technical points involved and it is also clear that we are dealing with structural properties, without unnatural assumptions. The paper deals at the same time both with divergence and nondivergence operators, showing that some properties are typical of elliptic equations per se, nothwithstanding their kind. Before coming to the full proof of the main result, a nice sketch of the main ideas is given, finally commenting upon what should be done in order to make everything rigorous (the actual proof is slightly different).

\section{Acknowledgments}

In concluding the work for this special issue, all the editors would like to thank the authors for their interesting contributions, the BVP editor-in-chief, prof. Ravi P. Agarwal, for the unique opportunity offered, and the Editorial Office of BVP for the superb support that has been provided during all the preparation.

Emmanuele DiBenedetto: Department of Mathematics, Vanderbilt University, 1326 Stevenson Center, Nashville, TN 37240, USA

Email address: em.diben@vanderbilt.edu

Ugo Gianazza: Dipartimento di Matematica “F. Casorati”, Università di Pavia, Via Ferrata 1,

27100 Pavia, Italy

Email address: gianazza@imati.cnr.it

Mikhail Safonov: School of Mathematics, University of Minnesota, 231 Vincent Hall, Minneapolis, MN 55455, USA

Email address: safonov@math.umn.edu 
José Miguel Urbano: Departamento de Matemática, Universidade de Coimbra, 3001-454 Coimbra, Portugal

Email address: jmurb@mat.uc.pt

Vincenzo Vespri: Dipartimento di Matematica “U. Dini”, Università di Firenze, Viale Morgagni 67/A, 50134 Firenze, Italy

Email address: vespri@math.unifi.it 